\title{
The morphological pathway for mouse forestomach cancer
}

\author{
GEORGE KOSSOY ${ }^{1}$, HERZL BEN-HUR ${ }^{2}$, ASHER ELHAYANY ${ }^{3}$, \\ DAVID F. SCHNEIDER ${ }^{4}$ and ITSHAK ZUSMAN ${ }^{1}$
}

\author{
${ }^{1}$ Koret School of Veterinary Medicine, Faculty of Agricultural, Food and Environmental Quality Sciences, The Hebrew \\ University of Jerusalem, Rehovot; ${ }^{2}$ Laboratory of Experimental Medicine, Park Rabin, Rehovot; ${ }^{3}$ Central District, Clalit \\ Health Services, Rishon Leziyon; ${ }^{4}$ Department of Obstetrics and Gynecology, Assaf Harofeh Medical Center, Tserifin, Israel
}

Received July 25, 2005; Accepted September 20, 2005

\begin{abstract}
We analyzed the morphological changes accompanying the development of cancer in the mouse forestomach. The main aim of the study was to evaluate whether cancer in this area of the stomach arises de novo or undergoes a series of precancerous changes. Tumors were induced by the 1,2-dimethylbenz $(a)$ antracene (DMBA) at a total dose of $4 \mathrm{mg} / \mathrm{mouse}$. The suspected areas of the stomach were studied morphologically in 79 mice. Benign tumors (squamous-cell papillomas) and malignant tumors (squamouscell carcinomas) were found in 40 mice. Tumors arose in all cases together with differential changes in the forestomach epithelium. These changes were seen as irregular diffuse hyperplasia or focal proliferation, with or without differential signs of dysplasia. Destruction of the basal epithelial membrane indicated transformation of the process into malignant invasive carcinoma. Thus, in chemically induced cancer of the forestomach, squamous-cell carcinoma develops as the final stage of morphologically recognizable precancerous changes in the epithelial layer. De novo formation of such tumors in the forestomach was not observed.
\end{abstract}

\section{Introduction}

It is well known that, in many cases, malignant tumors develop from so-called precancerous changes. With respect to the squamous epithelium of the skin, Shabad (1) regarded such changes as preneoplastic, diffuse or focal, circumscribed hyperplasia, followed by benign and then malignant tumors. Later on, the same sequence was described in the development of cancer of the urinary bladder epithelium (2). It has also been found that focal hyperplasia can show a very irregular structure $(3,4)$. The detailed description of these changes

Correspondence to: Professor I. Zusman, The Koret School of Veterinary Medicine, The Hebrew University of Jerusalem, P.O. Box 12, Rehovot 76100, Israel

E-mail: zusmani@agri.huji.ac.il

Key words: cancer, forestomach, mice resulted in the appearance of such terms as dysplasia and carcinoma in situ $(5,6)$. Thus, the process of carcinogenesis is currently described by the following morphological changes: diffuse hyperplasia $\rightarrow$ focal proliferation and metaplasia $\rightarrow$ benign tumor $\rightarrow$ grade I, II or III dysplasia $\rightarrow$ carcinoma in situ $\rightarrow$ invasive carcinoma. Because it has also been shown that any of these stages can be absent, the question is whether such a scheme is universal. Experimental (7) and clinical (8-10) observations have shown that glandular cancer can appear without visible precancerous changes. The de novo appearance of colorectal carcinoma in situ has been shown experimentally in rats (11) and clinically confirmed through advances made in endoscopy.

In humans, the endoscopic discovery of very small tumors without adenomatous elements nearby or any precursor lesions has led some physicians to the opinion that these tumors appeared de novo (12). Japanese and Western pathologists use different criteria to diagnose colorectal cancer. In Japan, colorectal carcinoma is diagnosed on the basis of nuclear and cellular patterns, even in cases considered by Western pathologists as non-invasive lesions with low-grade dysplasia. The Japanese approach promotes a higher rate of discovery of the early stages of colorectal carcinomas than in Western countries (13). These studies showed that de novo-appearing carcinomas have most, if not all, of the same molecular and structural defects generally found in colorectal carcinomas (9). It has been found that an adenomatous stage is part of the pathway in such tumors (14).

In trying to elucidate the question of whether cancer in the squamous epithelium of the digestive tract arises de novo or follows a series of precancerous changes, we analyzed the morphological pathway of carcinomas in the mouse forestomach lined with the squamous epithelium.

\section{Materials and methods}

Animals. All experiments were carried out in accordance with the guidelines established by the Institutional Animal Care and Use Committee of the Hebrew University of Jerusalem. Ninety-two 8-week old female mice of three different lines (Balb/c, FVB/N and C3H/He; Harlan Labs, Rehovot, Israel) were housed in polypropylene cages under a 14-/10-h light/ dark regime at $22 \pm 2^{\circ} \mathrm{C}$, and were provided standard lab chow and tap water ad libitum. 
A

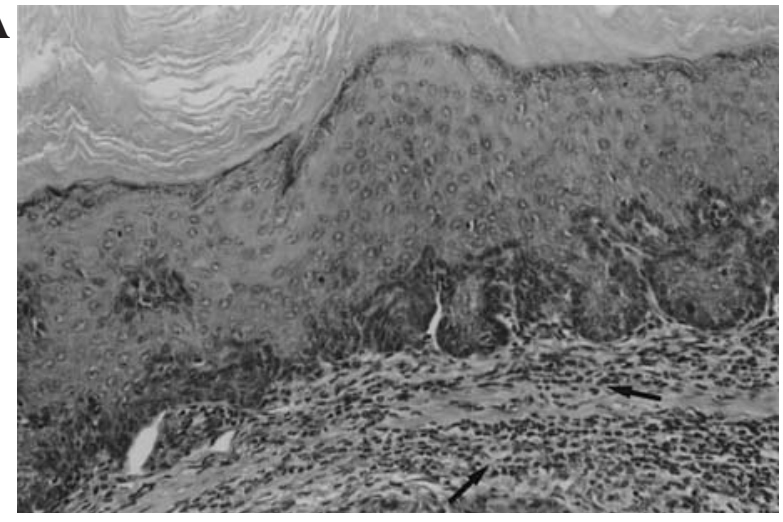

C

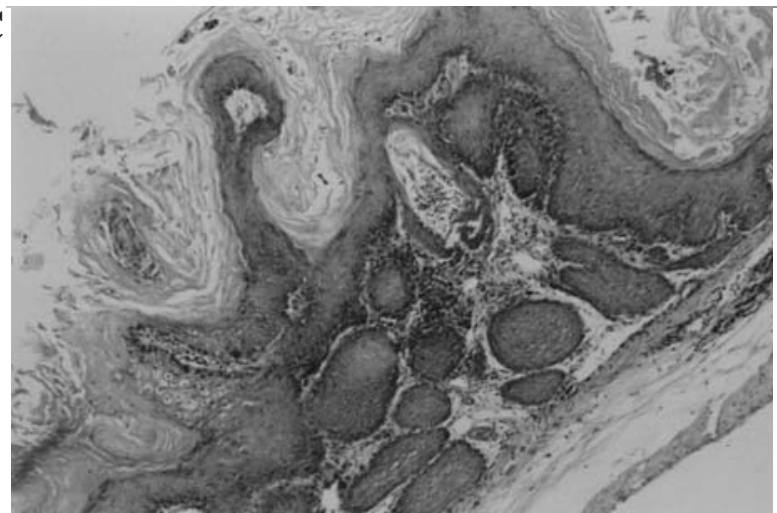

$\mathbf{E}$

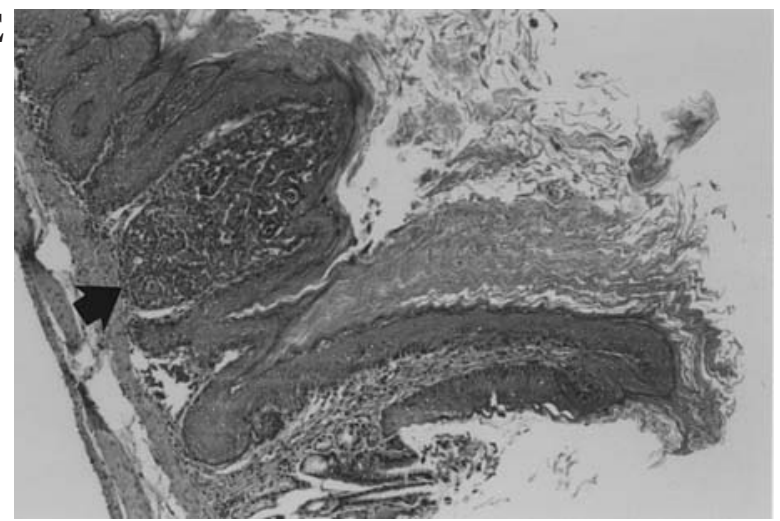

B

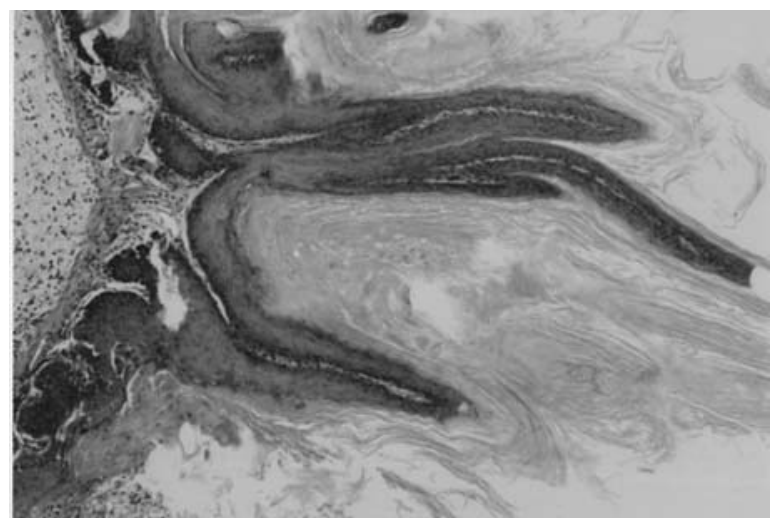

D

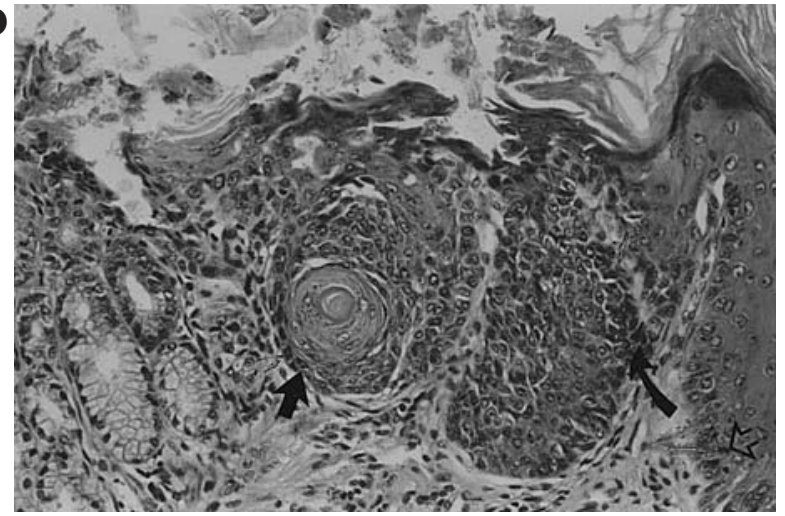

F

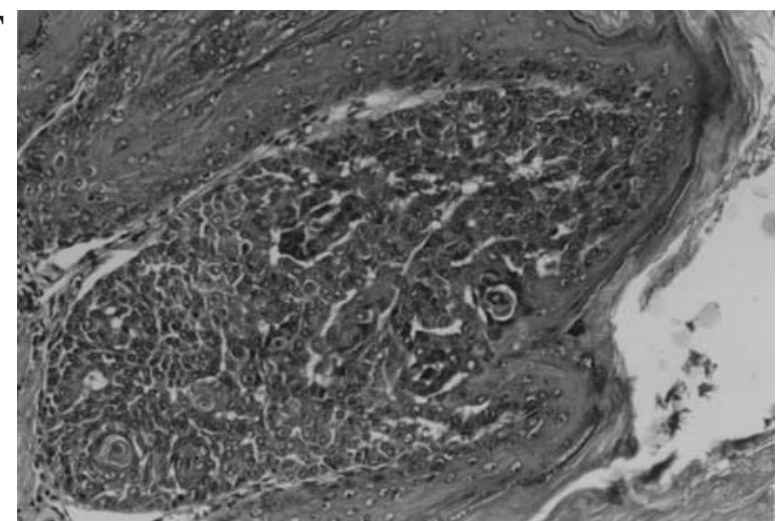

Figure 1. The mouse forestomach. Preinvasive changes of the squamous epithelium. H\&E. A-E, x80; F, x800. A, diffuse hyperplasia of the squamous epithelium. Note lymphoid infiltration in the subepithelial layer (arrows). B, squamous-cell papilloma against a background of diffuse irregular hyperplasia of the epithelium. C, squamous-cell papilloma against a background of focal proliferations. D, focal proliferations with signs of dysplasia: central hyperkeratosis (short arrow), focal nuclear hyper-chomatism (curve arrow), mitosis in the parabasal layer (white arrow). E and F, carcinoma in situ (arrow). Note integrity of the basal membrane in $\mathrm{E}$ and changed epithelial polarity, and cellular and nuclear polymorphism in $\mathrm{F}$.

Tumorigenic experiments. Tumors were induced with 9,10dimethyl-1,2-benz(a)anthracene (DMBA; Sigma, St. Louis, MO). Each animal received two intragastric feedings of DMBA, at a 1-week interval, at a dose of $2 \mathrm{mg}$ dissolved in $0.5 \mathrm{ml}$ corn oil. The total amount of carcinogen was $4 \mathrm{mg}$ / mouse.

Scoring and analysis. All mice were checked weekly for the appearance of tumors in the peritoneal cavity. Mice were killed by cervical dislocation, and all organs were checked for the presence of tumors.

Morphological analysis. The stomach was opened alongside the greater curvature and unwound. The mucous layer was analyzed for the presence of tumors. The affected parts of the stomach were fixed in $4 \%(\mathrm{v} / \mathrm{v})$ formaldehyde. All histological studies were performed using standard procedures with uniform conditions of fixation and staining of $3-\mu \mathrm{m}$ sections with hematoxylin and eosin (H\&E).

\section{Results}

Analysis of the mucous layer of the stomach in tumorbearing mice showed morphological changes only in the area of the forestomach, especially at the border with the glandular part of the stomach, and increasing toward the area of the limiting ridge. In the glandular epithelium, macroscopic changes were not found. 
Table I. Effects of DMBA on the rate of tumorigenesis in the mouse forestomach.

\begin{tabular}{lccccc}
\hline Mouse strain & Initial no. of mice & Effective no. of mice & & \multicolumn{2}{c}{ No. of mice with tumors in the forestomach } \\
\cline { 5 - 6 } & & & Total & Benign tumors & Malignant tumors \\
\hline Balb/c & 28 & 24 & 9 & 5 & 4 \\
FVB/N & 20 & 17 & 11 & 7 & 4 \\
C3H/He & 44 & 38 & 20 & 14 & 6 \\
Total & 92 & 79 & 40 & 26 & 14 \\
\hline
\end{tabular}

${ }^{a}$ The number of remaining mice when the first tumor appeared in a group.

A

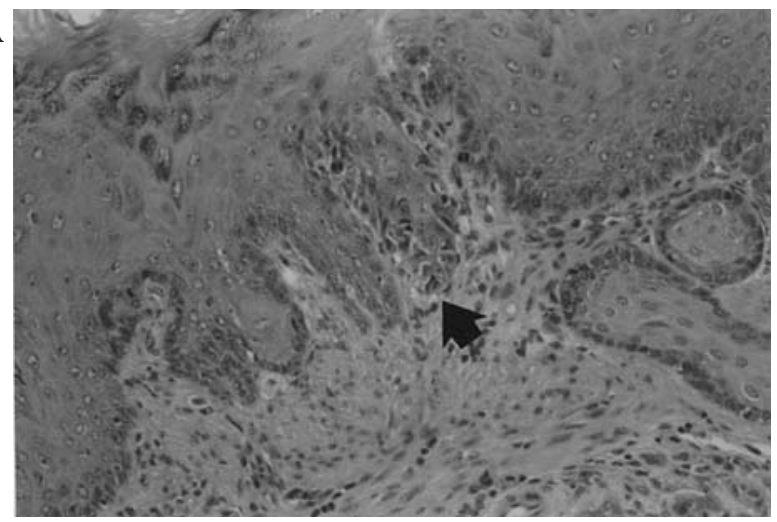

C

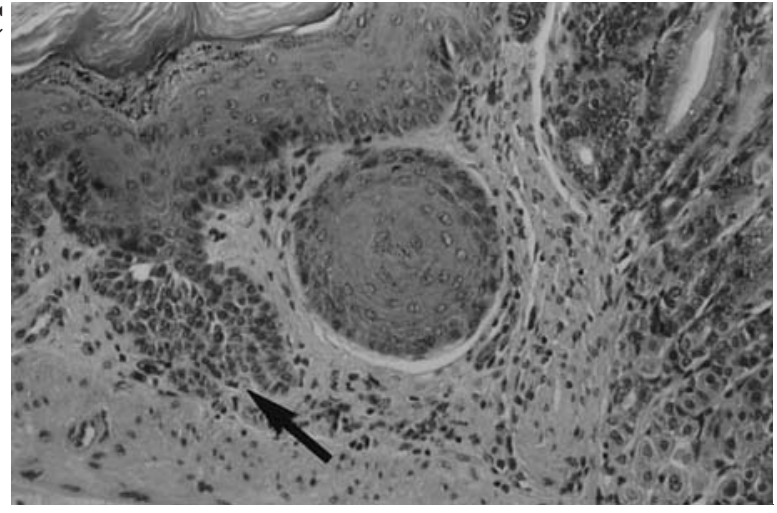

E

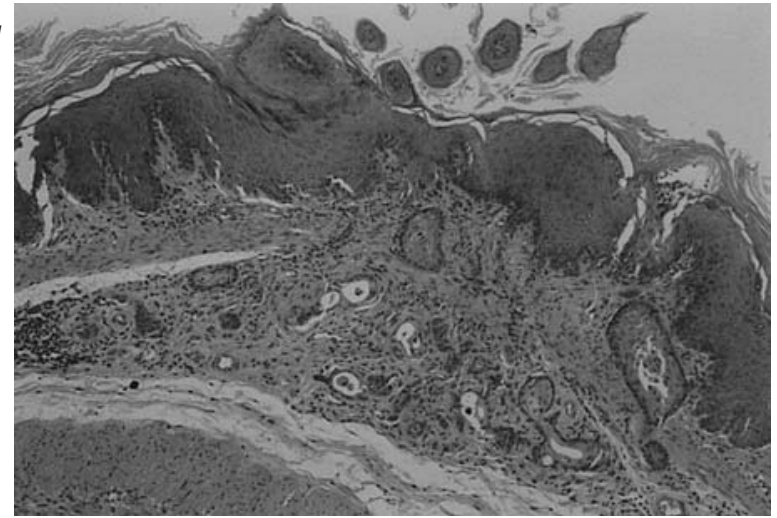

B

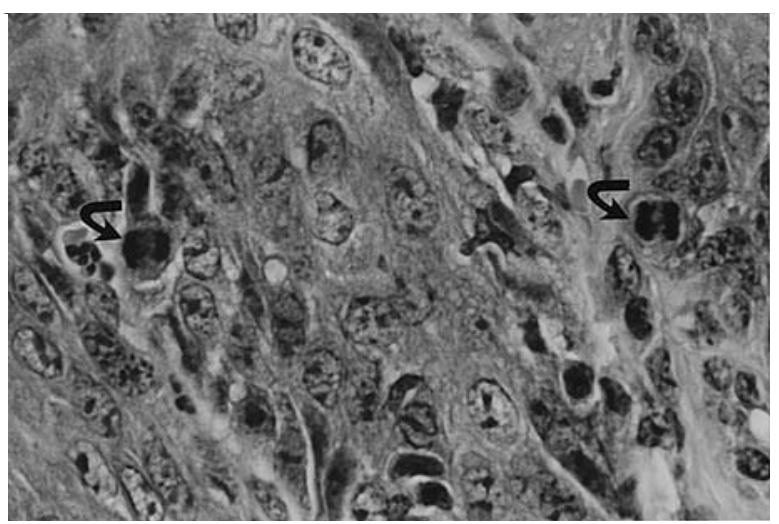

D

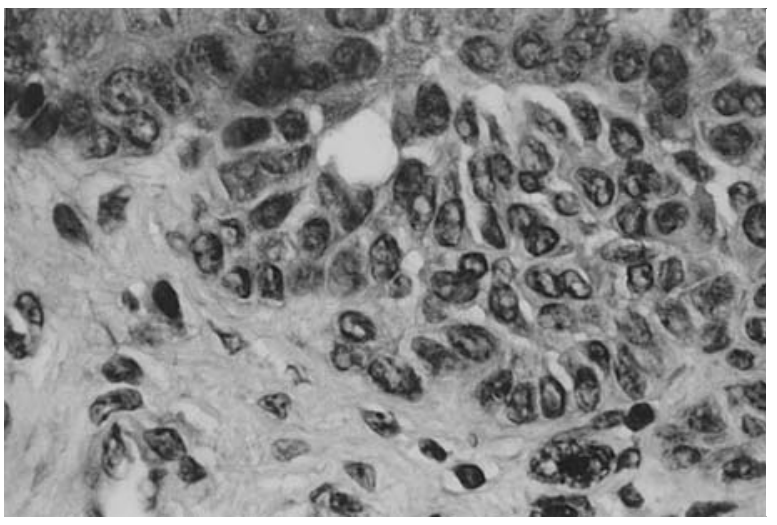

F

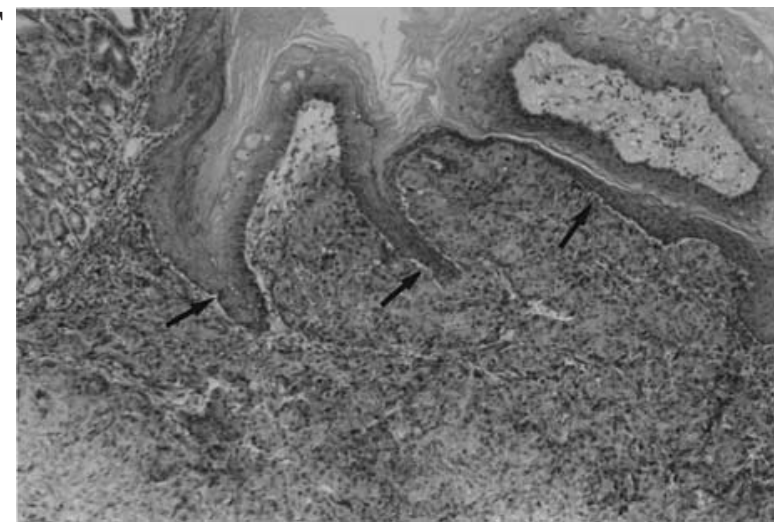

Figure 2. The mouse forestomach. Invasive changes of the squamous epithelium. H\&E. A and C, x200; B and D, x800; E and F, x80. A and B, small focal proliferations with areas of increased mitotic activity in the basal layer and the poorly defined basal membrane (arrow). Note the cells with mitotic figures (curve arrows). C and D, the disrupted basal membrane and cellular microinvasion into the subepithelial layer (arrow). Note the cellular and nuclear polymorphism in D. E, invasive squamous-cell carcinoma. Invasions present only in the subepithelial layer. Lymphoid infiltration is no longer seen. F, invasion of the forestomach wall with squamous-cell carcinoma without disruption of the epithelial layer (arrows). 
In the different mouse lines, the forestomach tumors had very similar structures (Table I). The benign tumors were represented by squamous-cell papillomas, whereas the malignant tumors were squamous-cell carcinomas with different levels of epithelial keratinization and early ulceration. Large tumors had deformed the stomach wall and were growing into neighboring organs. In all cases, the tumors developed following a series of differential changes in the epithelium.

The early changes were recognized as diffuse irregular hyperplasia of the epithelium with no visible defects in its structure (Fig. 1A). Lymphoid infiltration of the subepithelial layer is characteristic at this stage, reflecting the change in relationship between the parenchymal and stromal parts of the stomach wall.

Intensification of the process was reflected in enhancement of basal-cell hyperplasia and formation of focal proliferations. These changes were found to accompany the appearance of papillomas. The latter appeared together with either diffuse irregular epithelial hyperplasia (Fig. 1B) or focal proliferations in the basic parts of the papillomas (Fig. 1C). Prognoses are usually based on focal proliferations. Malignant transformation of the papillomas was not seen. Overall, the exofitic character of tumor growth more closely represented the benign process.

Intensification of dysplasia in areas with focal proliferations was characterized by disruption of the vertical anisomorphology of the epithelial layer, signs of hyper- and parakeratosis, basal-cell hyperplasia, nuclear hyperchromatism, nuclear polymorphism, enhancement of mitotic activity and appearance of individual mitosis in the parabasal layer (Fig. 1D). These features became enhanced with the progression of dysplasia. In carcinoma in situ, the change in epithelial polarity reached its peak (Fig. 1E and F).

Invasive carcinoma is characterized by disruption of the integrity of the basal membrane and microinvasions of proliferating basal cells into the subepithelial layer. Cells in these areas were characterized by nuclear polymorphism and high mitotic activity (Fig. 2A-D). After the transformation of tumors into invasive carcinomas, lymphoid infiltration was markedly reduced in the subepithelial layer (Fig. 2E).

The integrity of the basal membrane is the only trustworthy criterion to differentiate between noninvasive and invasive tumors. The basal membrane prevents both cellular growth into the subepithelial layer and the reverse invasive growth of a subepithelial malignant tumor into intact areas of the epithelial layer (Fig. 2F).

\section{Discussion}

Numerous, multiple genetic and epigenetic alterations are involved in the multistage process of carcinogenesis in different organs. The specific combination of alterations varies in different organs, as well as in different types of cancer. It has been shown, for example, that colorectal cancer can arise in one of two ways. In 70-80\% of cases, this cancer arises directly from the normal mucosal layer (de novo carcinoma), and in $20-30 \%$ of cases, the cancer has been described to result from a series of stages, from hyperplasia through adenoma to carcinoma in situ. Despite the fact that, in numerous studies, de novo carcinoma has been found to have mostly the same molecular defects as gradually developing carcinoma, different clinical and pathological parameters have been described in these two types of cancer (15).

Colorectal tumors are assumed to cover a wide range of premalignant and malignant lesions, such as uncontrolled epithelial-cell replication, dysplasia, adenomas of various dimensions and malignant adenocarcinomas $(4,16)$. In humans, many of these tumors can be removed by endoscopy. Experimental observations have shown that the pattern of carcinogenesis in the rat colon depends on the length of exposure to a carcinogen (17). This means that the term 'colorectal de novo carcinoma' certifies a furious rate of carcinogenesis but does not exclude its stages.

Another picture has been given to describe the origin of gastric cancer, which is characterized by two different histological types: well-differentiated or intestinal, and poorly differentiated or diffuse (10). The existence of these two types of cancer indicates that there are two distinct carcinogenetic pathways in gastric-cancer development. In well-differentiated cancer, three pathways can be distinguished: the intestinal metaplasia $\rightarrow$ adenoma $\rightarrow$ carcinoma in situ sequence, the intestinal metaplasia $\rightarrow$ carcinoma in situ sequence, or carcinoma de novo. The process is accompanied by molecular changes, such as loss of heterozygosis (LOH) and abnormal expression of the p73 gene, which is responsible for the development of alveolar gastric cancer. Poorly differentiated gastric cancer is characterized by LOH on chromosome $17 \mathrm{p}$, and $\mathrm{LOH}$ or mutation of the p53 gene and E-cadherin (10).

The biology of squamous-cell proliferation in the forestomach, as well as in other parts of the stomach, is not clearly understood. Morphological studies show that the tumorous lesions may follow a progression that starts with hyperplasia, the behavior of which is unpredictable. Some hyperplasias may develop into papillomas, whereas others may progress through dysplasia to squamous-cell carcinomas (18). Studies of chemically induced cancers have shown that cellular atypia may precede the squamous-cell carcinomas $(19,20)$. There is evidence that the genetic mechanism for chemically induced carcinoma in the rat forestomach is different in cancer caused by gene-toxic vs. non-toxic carcinogens (21). This difference is manifested in the differential frequency of alterations in genes such as H-ras and p53 by these different carcinogens.

Our study shows that chemically induced tumorigenesis in the mouse forestomach lined with squamous epithelium is a multistage process which is similar to that described for carcinogenesis in other types of squamous epithelium (18). This process consists of several phases, starting with diffuse inconsistent hyperplasia or focal proliferation, followed by the appearance of papillomas and adenomas, and their transformation into carcinoma in situ. Disruption of the basal membrane is the critical factor in the development of invasive carcinoma.

\section{References}

1. Shabad LM: Methodology of the study of carcinogens in human populations. J Toxicol Environ Health 4: 637-643, 1978.

2. Boorman GA, Wood $M$ and Fukushima S: Tumours of the urinary bladder. IARC Sci Public 111: 383-406, 1994.

3. Finckh ES: Aberrant, variant and deviant differentiation in pathology. Pathology 14: 443-447, 1982. 
4. Garcia SB, Park HS, Novelli M and Wright NA: Field cancerization, clonality, and epithelial stem cells: the spread of mutated clones in epithelial sheets. J Pathol 187: 61-81, 1999.

5. Misdraji J and Lauwers GY: Gastric epithelial dysplasia. Semin Diagn Pathol 19: 20-30, 2002.

6. Vindigni B and Galgani P: Gastric dysplasia: classification and clinical characteristics. Minerva Gastroenterol Dietol 50: 331-338, 2004.

7. Krutovskikh VA and Turusov VS: Tumours of the intestines. IARC Sci Publ 111: 195-221, 1994.

8. Yashiro M, Carethers JM, Laghi L, Saito K, Slezak P, Jaramillo E, Rubio C, Koizumi K, Hirakawa K and Boland CR: Genetic pathways in the evolution of morphologically distinct colorectal neoplasms. Cancer Res 61: 2676-2683, 2001.

9. Mueller JD, Bethke B and Stolte M: Colorectal de novo carcinoma: a review of its diagnosis, histopathology, molecular biology, and clinical relevance. Virchows Arch 440: 453-460, 2002.

10. Tahara E: Genetic pathways of two types of gastric cancer. IARC Sci Publ 157: 327-349, 2004.

11. Pozharisski KM: Pathology of tumours in laboratory animals. Tumours of the rat. Tumours of the intestines. IARC Sci Publ 99: 159-198, 1990.

12. Kuramoto $\mathrm{S}$ and Oohara T: Minute cancers arising de novo in the human large intestine. Cancer 61: 829-834, 1988.

13. Schlemper RJ, Itabashi M, Kato Y, Lewin KJ, Riddell RH, Shimoda T, Sipponen P, Stolte M and Watanabe H: Differences in the diagnostic criteria used by Japanese and Western pathologists to diagnose colorectal carcinoma. Cancer 82: 60-69, 1998.
14. Kuramoto S and Oohara T: How do colorectal cancers develop? Cancer 75 (suppl 6): 1534-1538, 1995.

15. Nakamura K: De novo cancer and adenoma-carcinoma sequence of the colorectum - clinicopathological differences between de novo carcinoma and carcinoma with the sequence. Nippon Geka Gakkai Zasshi 100: 766-775, 1999.

16. Ponz de Leon M and di Gregorio C: Pathology of colorectal cancer. Dig Liver Dis 33: 372-388, 2001.

17. Endo T, Ookawa K, Tanaka M, Nakaji S, Tsuchida S and Sugawara K: Differences in carcinogenesis by the length of carcinogen exposure period in rat colon. Dig Dis Sci 46: 109-117, 2001.

18. Leininger JR and Jokinen MP: Tumours of the oral cavity, pharynx, oesophagus and stomach. IARC Sci Publ 111: 167-193, 1994.

19. National Toxicology Program: NTP toxicology and carcinogenesis studies of 2,4-hexadienal (89\% trans, trans isomer, CAS no. 142-83-6; $11 \%$ cis, trans isomer) (Gavages Studies). Natl Toxicol Prog Tech Rep Ser 509: 1-290, 2003.

20. Doi AM, Irwin RD and Bucher JR: Influence of functional group substitutions on the carcinogenicity of anthraquinone in rats and mice: analysis of long-term bioassays by the National Cancer Institute and the National Toxicology Program. J Toxicol Environ Health B Crit Rev 8: 109-126, 2005.

21. Kaneko M, Morimura K, Nishikawa T, Wanibuchi H, Takada N, Osugi H, Kinoshita H and Fukushima S: Different genetic alterations in rat forestomach tumors induced by genotoxic and non-genotoxic carcinogens. Carcinogenesis 23: 1729-1735, 2002 . 